\title{
The Automatic Velocity Evolution Model Of Space Debris
}

\author{
Bin Ren ${ }^{1, a}$ \\ ${ }^{1}$ School of North China Electric Power University, Baoding 071003, China; \\ a1624352537@qq.com
}

Keywords: collision, Reynolds's study, simulation

\begin{abstract}
Space is becoming cluttered with litter. For nearly fifty years, people have been launching rockets into Space, in orbit around Earth, and beyond. The remains of derelict satellites and rockets and the debris resulting from their explosions and collisions with one constitute a significant fraction of the objects in space-far larger in number and mass than spacecraft that are still operational. We set up the automatically velocity evolution model of space debris to predict the speed of space debris.
\end{abstract}

\section{Introduction}

With the continuous development of aerospace science and technology, mankind has developed the history of the space time and time again. However, the space junk, meanwhile, is growing over time. For example, the rockets arrows body, the disabled spacecraft, the task-related debris, and the broken pieces, etc. The debris ranges in size and mass. It is estimated that more than 500,000 pieces of space debris, also called orbital debris, are currently being tracked as potential hazards to space craft. When the Russian satellite Kosmos-2251 and the USA satellite Iridium-33 collided on 10 February, 2009, it destroyed both satellites and created a field of debris that endangers other orbiting satellites. If we don't control or adopt measures to remove timely, the situation will become more dangerous.

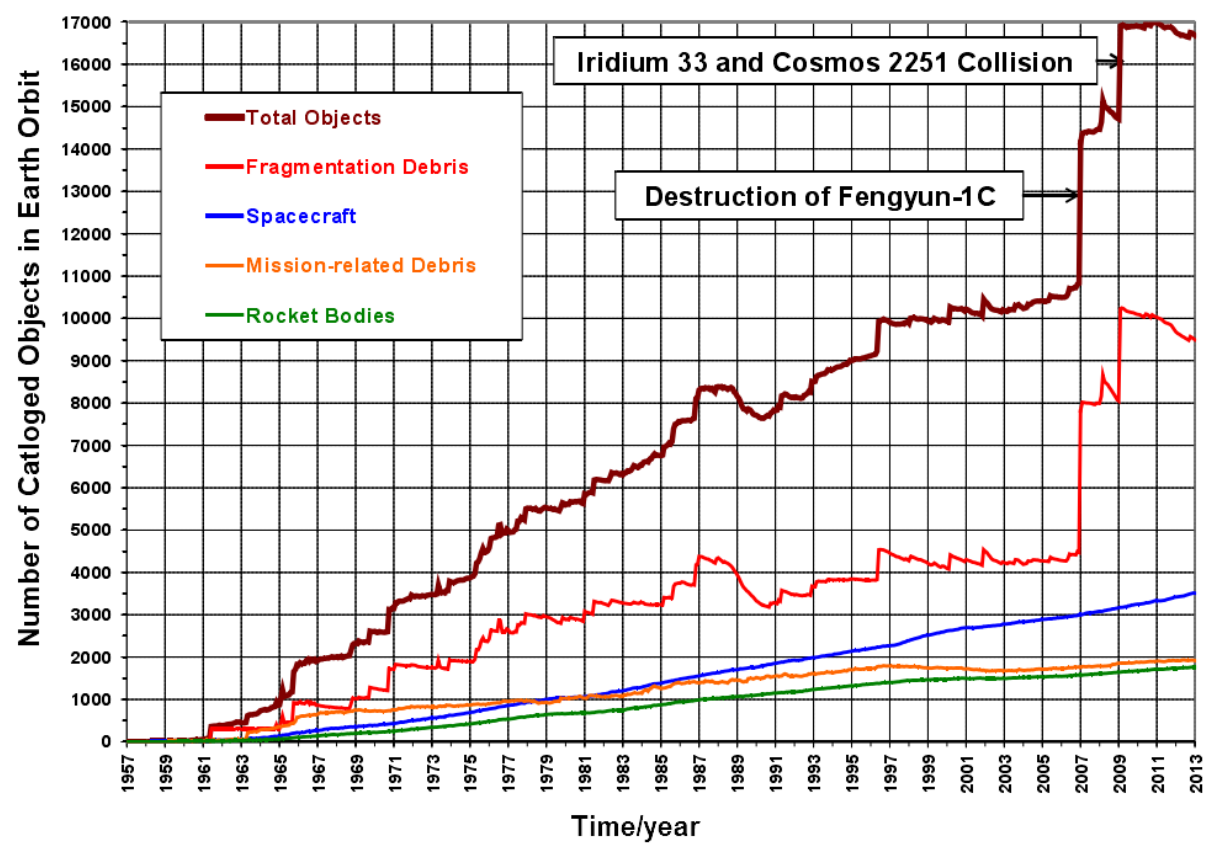

Figure 1.Numble of cataloged objects in Earth orbit

Through consulting relevant literature, during the current solution for space junk, the relatively perfect theories are the following kinds, laser removal technology, satellite debris collector, space garbage trucks, suicide satellite, and resistance-increase device.

- We hope to find a right way (or a combination) from these solutions through reasonable analysis, which can clean up the existing space junk.

- Establish a mathematical model of space debris over time and get the evolution of debris. 
- Now, most of the technological approaches have not become funded projects and there is no business plan extant to reduce the amount of space debris.

Our model considers qualitative and/or quantitative estimates of the costs, risks, benefits, and other important factors.

- Using our model to determine whether an economically attractive opportunity exists or no such opportunity is possible. If a viable commercial opportunity exists as an alternative solution, provide a comparison of the different options for removing debris, and include a specific recommendation as to how the debris should be removed. If no such opportunity is possible, then provide innovative alternatives for avoiding collisions.
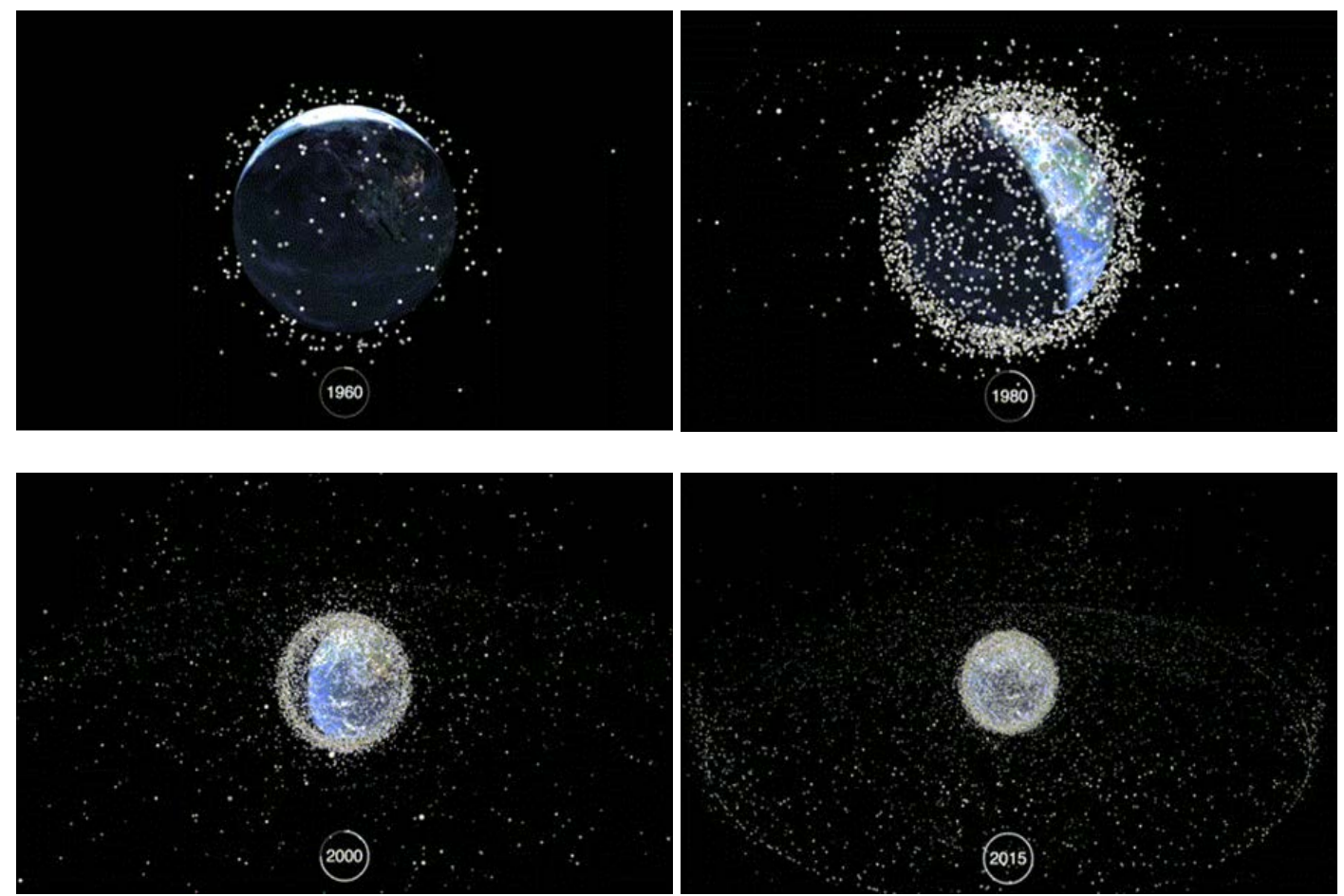

Figure 2

\section{Velocity Distribution Model}

There will be incremental velocity of debris after collision in different directions. The increment of speed can be used with the following formula to calculate the increment.

Velocity distribution model is to describe freshmen debris velocity increment when later collision occurs, and to determine the freshmen pieces of track. Then it provides a basis for the next evolution of the debris.

According to Reynolds's study, speed increments in the explosion:

$$
\lg (\overline{\Delta v})=-0.0676(\lg d)^{2}-0.804(\lg d)-1.514
$$

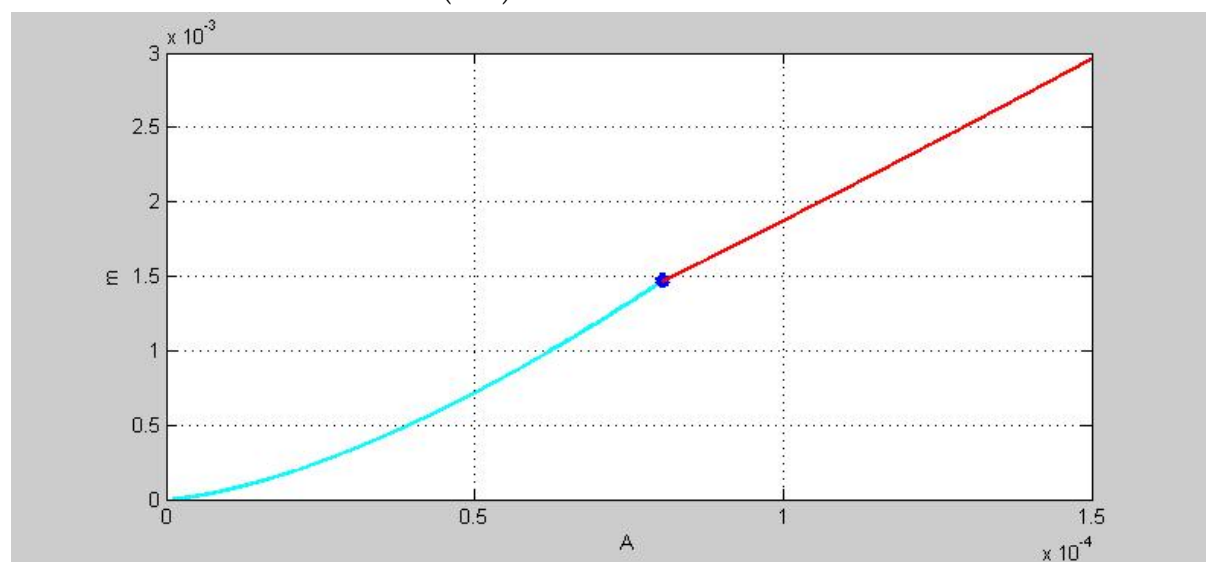

Figure 3 
From this curve we can see that with the increase of the sectional area, the quality of debris increase more quickly. But when it reaches a certain critical value, the tendency is slow down.

It reasonable to express the reflect of ratio of area and mass to space junks' movements.

According to Su's study, the velocity increments in the collision are,

$$
\lg \left(\frac{\overline{\Delta v}}{v_{i}}\right)= \begin{cases}-0.125-0.0676 \times \lg \left(\frac{d}{d_{m}}\right) & d>d_{m} \\ -0.125 & d \leq d_{m}\end{cases}
$$

In which, $d_{m}=\frac{\sqrt[3]{E_{p}}}{C}, E_{p}=\frac{v^{2} m_{p}}{2},{ }^{v_{i}}$ represents the relative collision speed, $m_{p}$ is the quality collision, $v_{p}$ is the speed of impactors, $C=8.01 \times 10^{8}$.

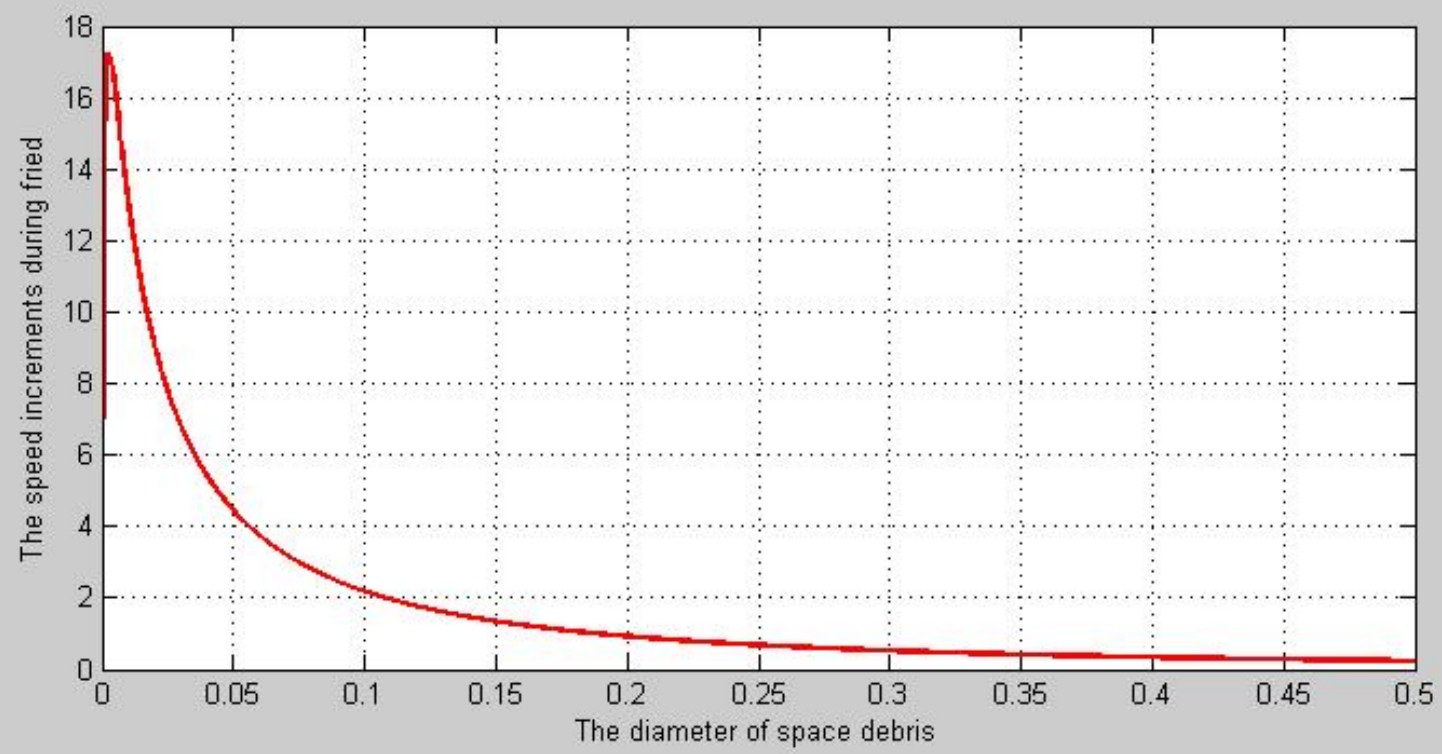

Figure 4

After checking the related data we can get the DFH-1 satellite equivalent diameter to be $0.5 \mathrm{~m}$, we change the diameter of the debris until it reaches the satellite's quality to get the curve. We can clearly see from the above curve when the debris is small, the quality of the resulting explosion velocity increment will increase rapidly, and when a particular value is obtained in the detonation velocity increment will reach the peak. While the quality of the debris is relatively small and with the increase of the quality of the debris, the velocity increment has been slow.

After obtaining, the true size of the debris speed increment can be drawn triangular distribution obtained by Jehn,

$$
\Delta v= \begin{cases}\overline{\Delta v}(0.1+0.6 \sqrt{3 y}) & 0 \leq y<0.75 \\ \overline{\Delta v}(1.3-0.6 \sqrt{1-y}) & 0.75 \leq y<1\end{cases}
$$




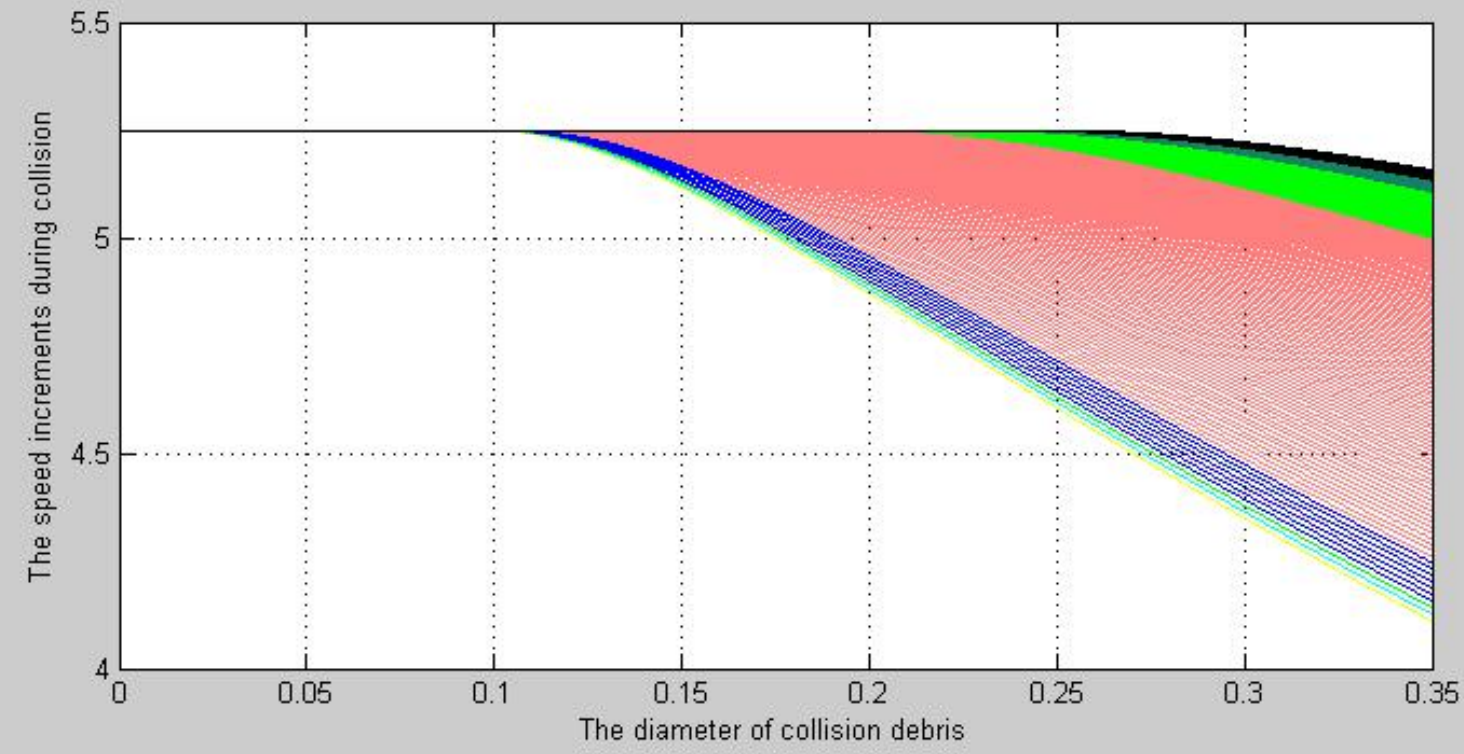

Figure 5

We assume that the relative impact velocity is $7 \mathrm{~km} / \mathrm{s}$, through the collision debriss of maximum diameter changes, to get the curve, we can clearly see from the above curve as the change of the maximum diameter of collision debriss, in the process of collision velocity increment as the change of the diameter of the collision debriss curve upward as a whole.

\section{References}

[1] https://en.wikipedia.org/wiki/Space_debris\#Dealing_with_debris

[2] https://en.wikipedia.org/wiki/Stackelberg_competition

[3]https://www.nasa.gov/press-release/nasa-confirms-evidence-that-liquid-water-flows-on-today-smars

[4]Taylor M W. Orbital debris: Technical and legal issues and solutions[D]. McGill University, 2006.

[5]Macauley M K. The economics of space debris: Estimating the costs and benefits of debris mitigation[J]. Acta Astronautica, 2015, 115: 160-164. 